\title{
Algorithm-aided diagnosis of chronic pulmonary aspergillosis in low- and middle-income countries by use of a lateral flow device
}

\author{
Richard Kwizera ${ }^{1,2} \cdot$ Andrew Katende $^{3} \cdot$ Anneth Teu $^{4} \cdot$ Denise Apolot ${ }^{4} \cdot$ William Worodria ${ }^{3} \cdot$ Bruce J. Kirenga $^{2,3}$. \\ Felix Bongomin $^{3,5}$ (D)
}

Received: 28 November 2019 / Accepted: 28 November 2019 / Published online: 6 December 2019

(C) Springer-Verlag GmbH Germany, part of Springer Nature 2019

Chronic pulmonary aspergillosis (CPA) is a slowly progressive parenchymal lung disease typically caused by Aspergillus fumigatus [1]. CPA affects immunocompetent or subtly immunocompromised patients with underlying structural lung diseases [2] and is estimated to affect approximately three million people per year worldwide [3]. It can co-exist with pulmonary tuberculosis (PTB), has both pulmonary and systemic symptoms that are clinically indistinguishable from that of PTB, and is often misdiagnosed and managed as smearnegative PTB [4]. According to the Infectious Diseases Society of America (IDSA), the European Society for Clinical Microbiology and Infectious Diseases (ESCMID), the European Confederation of Medical Mycology (ECMM), and the European Respiratory Society (ERS) Guidelines, the diagnosis of CPA should be based on characteristic symptoms and radiologic features present or presumed to have been present for at least 3 months in a patient with no or minimal immunosuppression and a prior or current lung condition with microbiological or immunological evidence of Aspergillus spp. infection [5]. This definition is consistent

Richard Kwizera and Andrew Katende are joint first authors.

Felix Bongomin

drbongomin@gmail.com

1 Infectious Diseases Institute, College of Health Sciences, Makerere University, Kampala, Uganda

2 Makerere University Lung Institute, College of Health Sciences, Makerere University, Kampala, Uganda

3 Department of Internal Medicine, Makerere University College of Health Sciences, Makerere University, Kampala, Uganda

4 Department of Radiology, Makerere University College of Health Sciences, Makerere University, Kampala, Uganda

5 Department of Medical Microbiology \& Immunology, Faculty of Medicine, Gulu University, Gulu, Uganda with the original definition of CPA proposed by Denning and colleagues [1]. Still, CPA is under- and mis-diagnosed in resource-constrained settings where adequate diagnostics are unavailable [6]. Previously treated PTB is the most common risk factor for the development of CPA even in the developed world [1]. The global burden of CPA attributed to healed TB lesions alone has been estimated to over 1.2 million cases annually globally [7]. On the other hand, active PTB is the number one differential diagnosis for CPA and CPA is the number one differential diagnosis for patients previously treated for microbiologically confirmed PTB who are currently sputum smear-negative [6]. Recent evidence has shown that the annual rate of new CPA development following completion of PTB treatment is about $6.5 \%$ in those with chest radiography cavitation and $0.2 \%$ in those without [8] (Fig. 1).

The diagnosis of CPA is based on a combination of clinical symptoms, compatible chest imaging findings, evidence of Aspergillus infection (including Aspergillus-specific IgG, precipitins, and mycological cultures of respiratory samples) or histology, and the exclusion of alternative diagnosis [1]. Detection of Aspergillus-specific IgG is the most reliable evidence of CPA and has been its diagnostic cornerstone $[9,10]$. Aspergillus-IgG serology using ELISA has been the mainstay of immunological evidence of Aspergillus spp. infection in CPA [11]. ELISA instruments are expensive and labor and resource intensive; diagnostic cutoffs vary by ethnicity and technologies used which renders this tool not suitable for resource-limited laboratory settings. Obviously, this has been a major challenge in making a definitive diagnosis of CPA in resource-constrained settings.

A recent lateral flow device (LDBIO Diagnostics, Lyon, France), which has a run time of less than $30 \mathrm{~min}$, is simple to use, and requires minimal laboratory equipment, has consistently been shown to have a good sensitivity $(\sim 85-92 \%)$ and specificity ( 94-98\%) in multisite validation studies, making it a suitable diagnostic tool for the serological 

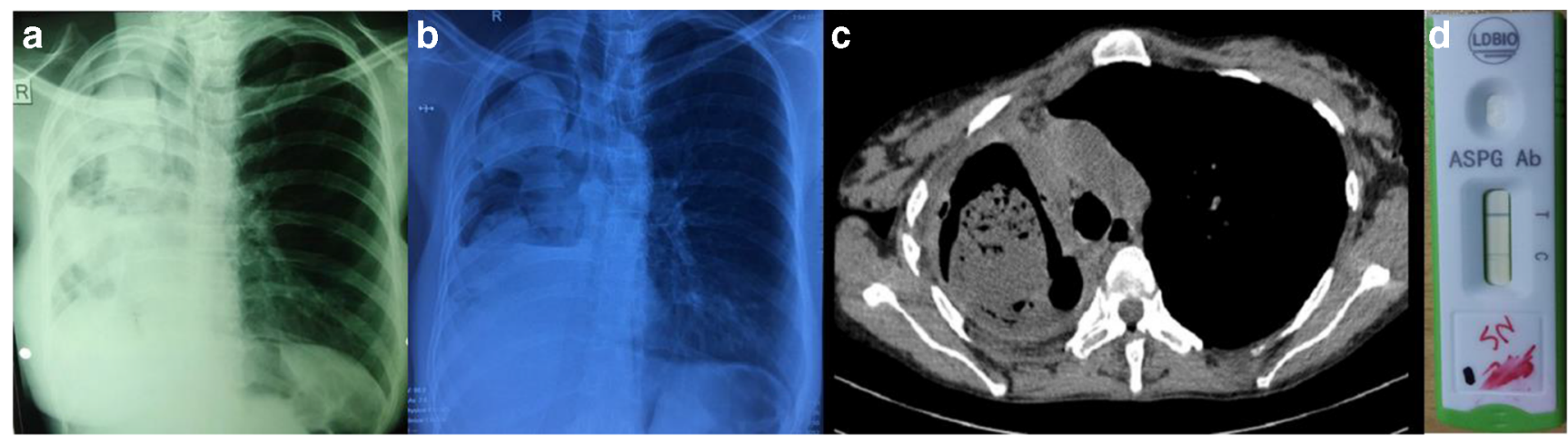

Fig. 1 a Chest x-ray from 2016 showing upper lobe cavities with fungal ball. b Chest x-ray from 2019 showing upper lobe cavities with fungal ball. c Contrasted chest CT scan from 2019 showing right upper lobe aspergilloma, right bronchopleural fistula with hydro-pneumothorax, and left cystic and varicose bronchiectasis. d Positive Aspergillus IgMIgG LFD (+++) diagnosis of CPA in LMIC [12]. As a consequence, simplified algorithms for the diagnosis of CPA in resource-constrained settings were published $[13,14]$ to permit early and correct diagnosis and appropriate management of the disease. In this algorithm, CPA was defined as "illness for $>3$ months and all of the following: (1) weight loss, persistent cough, and/or hemoptysis; (2) chest images showing progressive cavitary infiltrates and/or a fungal ball and/or peri-cavitary fibrosis or infiltrates or pleural thickening; and (3) a positive Aspergillus IgG assay result or other evidence of Aspergillus infection" and exclusion of TB infection. We have been able to better diagnose $\mathrm{CPA}$ in routine clinical practice in a resource-limited setting, an example of which is illustrated in Textbox 1. Over the past episode, we identified more than 20 otherwise undetected CPA cases using an algorithm including the lateral flow device (LFD).

Textbox 1 Illustrative diagnostic case

A 40-year-old Ugandan woman was referred to Pulmonology with a 3-month history of cough with mucopurulent and foul smelling sputum without blood. Her cough worsened 2 weeks prior to admission and was associated with pleuritic chest pain, difficulty in breathing, low-grade fevers, weight loss, and anorexia but no night sweats. She reported exertional dyspnea but no orthopnea or paroxysmal nocturnal dyspnea or lower limb swelling. She was on anti-TB drugs for a week prior to admission. She had no history of smoking or drinking alcohol. Other systems were essentially normal. Medical history showed HIV infection diagnosed 13 years ago with PTB as the index opportunistic infection. Since then, she had been on AZT/3TC/EFV for antiretroviral therapy (ART) with self-reported adherence. Her CD4 T cell count was 176 cells/mL. She had a blood pressure of $125 / 65 \mathrm{mmHg}$, respiratory rate of $20 \mathrm{bpm}$, pulse rate of $110 \mathrm{bpm}$, and $\mathrm{SpO}_{2}$ of $96 \%$ on room air. She was moderately pale with extensive oral thrush and a grade III digital clubbing. She had no palpable lymph nodes. Chest examination revealed a flattened right infra-clavicular region with tracheal deviation to the right without displacement of the point of maximum cardiac intensity. There was reduced chest movement (right, supra-mammary) with an increased tactile fremitus; dull percussion note and amphoric breathing were noted. She had a stony dull percussion note with absent breath sounds in the right infra-scapulary region. Cardiac examination revealed a tachycardia with normal heart sounds. Her chest X-rays showed upper lobe cavities with fungal balls and chest CT scan was consistent (Fig. 1). Complete blood count showed a leukocytosis (14,000 cells/mL) with neutrophilia $(81 \%)$ and macrocytic anemia of $8.0 \mathrm{~g} / \mathrm{dL}$. Sputum GeneXpert MTB/RIF ultra was negative for Mycobacterium tuberculosis and urine lipoarabinomannan (LAM) was negative. Renal and liver function tests were within normal limits and hepatitis B and C antibody tests were negative. However, Aspergillus-specific LFD (IgG-IgM) was strongly positive (Fig. 1). A diagnosis of advanced HIV with clinical and immunological failure, with post-TB CPA, AZT-induced macrocytic anemia, oral candidiasis, and bronchopulmonary fistula with hydro-pneumothorax was made. We discontinued anti-TB drugs and changed her ART regimen to TDF/3TC/DTG. We commenced her on itraconazole at a dose of $200 \mathrm{mg}$ twice daily and antibiotics for superimposed bacterial pneumonia. She received 2 units of packed red blood cells. She was in the hospital for 2 weeks, improved significantly, and was discharged on her new ART regimen and itraconazole.

In high-burden PTB nations, exclusion of active tuberculosis is the most important first step in the diagnosis of CPA. This is often not an issue, as highly sensitive pointof-care tests are widely available, such as the GeneXpert MTB/RIF PCR test [15]. However, if made widely available and accessible, Aspergillus LFD may change the way CPA is diagnosed in resource-limited settings. In addition, Aspergillus LFDs will contribute to anti-PTB stewardship, reducing the unnecessary prescription of toxic antibiotics and possibly controlling development of antibiotic-resistant PTB. Last but not least, resourcelimited settings are now open to participate in international CPA research since a definitive diagnosis of CPA can be achieved. We therefore advocate for Aspergillusspecific IgG LFD to be included on the WHO list of essential diagnostics. 
Acknowledgments Dr. Lydia Nakiyingi is gratefully acknowledged for patient care.

Authors' contributions FB and RK conceived the manuscript. FB, RK, and $\mathrm{AK}$ drafted the manuscript. FB, RK, AK, AT, AD, BJK, and WW contributed to the critical review of the manuscript.

Funding information RK is currently supported through the DELTAS Africa Initiative grant no. DEL-15-011 to THRiVE-2, from Wellcome Trust grant no. 107742/Z/15/Z and the UK government.

\section{Compliance with ethical standards}

Conflict of interest The authors declare that they have no conflict of interest.

Ethics The authors confirm that the ethical policies of the journal, as noted on the journal's author guidelines page, have been adhered to and the appropriate ethical review committee approval has been received.

\section{References}

1. Denning DW, Riniotis K, Dobrashian R, Sambatakou H (2003) Chronic cavitary and fibrosing pulmonary and pleural aspergillosis: case series, proposed nomenclature change, and review. Clin Infect Dis 37(Suppl 3):S265-S280. https://doi.org/10.1086/376526

2. Hayes GE, Denning DW (2013) Frequency, diagnosis and management of fungal respiratory infections. Curr Opin Pulm Med 19(3): 259-265. https://doi.org/10.1097/MCP.0b013e32835flad1

3. Bongomin F, Gago S, Oladele RO, Denning DW (2017) Global and multi-national prevalence of fungal diseases - estimate precision. J Fungi 3(4):57

4. Kwizera R, Parkes-Ratanshi R, Page ID, Sekaggya-Wiltshire C, Musaazi J, Fehr J et al (2017) Elevated Aspergillus-specific antibody levels among HIV infected Ugandans with pulmonary tuberculosis. BMC Pulm Med 17(1):149. https://doi.org/10.1186/ s12890-017-0500-9

5. Patterson TF, Thompson GR III, Denning DW, Fishman JA, Hadley S, Herbrecht R et al (2016) Practice guidelines for the diagnosis and management of aspergillosis: 2016 update by the
Infectious Diseases Society of America. Clin Infect Dis 63(4):e1e 60

6. Oladele R, Irurhe N, Foden P, Akanmu A, Gbaja-Biamila T, Nwosu A et al (2017) Chronic pulmonary aspergillosis as a cause of smearnegative TB and/or TB treatment failure in Nigerians. Int J Tuberc Lung Dis 21(9):1056-1061

7. Denning DW, Pleuvry A, Cole DC (2011) Global burden of chronic pulmonary aspergillosis as a sequel to pulmonary tuberculosis. Bull World Health Organ 89:864-872

8. Page ID, Byanyima R, Hosmane S, Onyachi N, Opira C, Richardson $\mathrm{M}$ et al (2019) Chronic pulmonary aspergillosis commonly complicates treated pulmonary tuberculosis with residual cavitation. Eur Respir J 53(3):1801184. https://doi.org/10.1183/ 13993003.01184-2018

9. Denning DW, Cadranel J, Beigelman-Aubry C, Ader F, Chakrabarti A, Blot $\mathrm{S}$ et al (2016) Chronic pulmonary aspergillosis: rationale and clinical guidelines for diagnosis and management. Eur Respir $\mathrm{J}$ 47(1):45-68

10. Page ID, Richardson M, Denning DW (2015) Antibody testing in aspergillosis - quo vadis? Medical mycology, myv020

11. Richardson M, Page I (2018) Role of serological tests in the diagnosis of mold infections. Curr Fungal Infect Rep 12(3):127-136. https://doi.org/10.1007/s12281-018-0321-1

12. Piarroux RP, Romain T, Martin A, Vainqueur D, Vitte J, Lachaud L et al (2019) Multicenter evaluation of a novel immunochromatographic test for anti-Aspergillus IgG detection. Front Cell Infect Microbiol 9:12. https://doi.org/10.3389/fcimb.2019.00012

13. Denning DW, Page ID, Chakaya J, Jabeen K, Jude CM, Cornet M et al (2018) Case definition of chronic pulmonary aspergillosis in resource-constrained settings. Emerg Infect Dis 24(8). https://doi. org/10.3201/eid2408.171312

14. Takazono T, Izumikawa K (2018) Recent advances in diagnosing chronic pulmonary aspergillosis. Front Microbiol 9:1810. https:// doi.org/10.3389/fmicb.2018.01810

15. Dorman SE, Schumacher SG, Alland D, Nabeta P, Armstrong DT, King B et al (2018) Xpert MTB/RIF Ultra for detection of Mycobacterium tuberculosis and rifampicin resistance: a prospective multicentre diagnostic accuracy study. Lancet Infect Dis 18(1): 76-84. https://doi.org/10.1016/S1473-3099(17)30691-6

Publisher's note Springer Nature remains neutral with regard to jurisdictional claims in published maps and institutional affiliations. 